\title{
Aspectos epidemiológicos y de análisis del síndrome de aborto bovino ${ }^{\#}$
}

\author{
Epidemiological and analytical aspects of bovine abortion syndrome
}

\author{
P Gädicke ${ }^{\mathrm{a}^{*}}$, G Monti ${ }^{\mathrm{b}}$ \\ aPrograma Doctorado en Ciencias Veterinarias, Facultad de Ciencias Veterinarias, Universidad Austral de Chile, Valdivia, Chile. \\ bInstituto de Medicina Preventiva Veterinaria, Facultad de Ciencias Veterinarias, Universidad Austral de Chile, Valdivia, Chile.
}

\section{SUMMARY}

\begin{abstract}
Bovine abortion is a limiting factor for the dairy sector, because it causes a decrease in milk production and the potential number of replacements of the herd, and also it constitutes a challenge for the veterinary profession. The objective of this paper is to review the epidemiology and different ways of analyzing the Bovine Abortion Syndrome (BAS). This review is divided in two parts; the first one presents an overview of the epidemiology, impact of the disease and risk factors; the second one presents some methodologies aimed to quantify and analyze BAS frequency and surveillance systems. Many differences can be found between the studies that analyze bovine abortion, the same happens with the way of calculating epidemiological indicators and definitions of important events. Also there are important differences regarding the subject in terms of presentation, methods of calculation of risk factors and especially the definition of events of importance. It is necessary to make a clear distinction between factors at animal and herd level, which could be associated with different production systems. In Chile, further investigation on BAS is needed in order to design more realistic and efficient control programs since risk factors reported in other countries could not necessarily be extrapolated to our local situation.
\end{abstract}

Palabras clave: aborto bovino, epidemiología, control.

Key words: bovine abortion, epidemiology, control.

\section{INTRODUCCION}

El aborto bovino es un factor limitante en el desarrollo ganadero en todos los países. Los casos esporádicos y los brotes epidémicos de abortos en vacas lecheras son un problema de creciente importancia que impacta significativamente en la productividad del rebaño al disminuir su viabilidad y desempeño productivo, al reducir el número potencial de vaquillas de reemplazo y la producción de leche, además de incrementar los costos asociados con la alimentación, tratamientos, inseminación y descarte prematuro de animales (Thurmond y Picanso 1990, Thurmond y col 1990 , Hovingh 2002).

Las pérdidas gestacionales se pueden producir en distintas etapas: en la de huevo (desde la concepción hasta el reconocimiento materno), embrión (reconocimiento materno hasta el final del período de diferenciación, alrededor del día 42) o feto (desde el día 42 al 260) (Fetrow y col 1990, Thurmond y Picanso 1990, Forar y col 1996, Rivera 2001). Las pérdidas que ocurren entre el día 260 hasta el término de la gestación se consideran partos prematuros, ya que el ternero estaría en condiciones de sobrevivir fuera del útero (Miller 1986, Dubovi 1994).

El objetivo de este artículo es revisar los principales aspectos relacionados con la epidemiología y formas de

\footnotetext{
Aceptado: 23.01.2008.

\# Financiamiento: DID-UACh Proyecto No S-2006-54.

* Picarte 3675, Valdivia, Chile; pgadicke@udec.cl
}

análisis poblacional del Síndrome de Aborto Bovino (SAB). Para esto el trabajo se organiza en dos partes: la primera incluye aspectos epidemiológicos, impacto y factores de riesgo; en la segunda se presentan algunas metodologías para su cuantificación, análisis y sistemas de vigilancia.

\section{PARTE 1}

\section{ABORTO BOVINO COMO SINDROME}

Síndrome se entiende como el conjunto de fenómenos que caracterizan una situación determinada. En el contexto del aborto bovino se necesita de una conjunción de varios factores para que se produzca la muerte fetal, lo que implica diferentes clases de mecanismos causales, los cuales pueden actuar en forma independiente o interactuando entre ellos (Rothman 2002). Además puede pasar un intervalo de tiempo largo entre la exposición a la causa del aborto y la observación del mismo (Miller 1986).

Existen varios estudios publicados tanto en Chile (Celedón y col 1998, Patitucci y col 2000, Sickles y col 2000, Reinhardt y col 2001, 2002, Paredes y col 2002, Ramírez y col 2002, Lertora y col 2003, Paredes y Moroni 2005) como en la literatura internacional (Bercovich 1998, Bolin 1998, McAllister y col 1998, Dubey 1999, Lindberg y Alenius 1999, Thurmond y col 2002) que informan de diferentes etiologías de aborto, especialmente las de tipo infeccioso, pero no existe suficiente información que trate el síndrome aborto bovino de manera integral, a pesar de 
que numerosos estudios demuestran su etiología multifactorial (Miller 1986, Gröhn y col 1990, Kirkbridge 1990, Jamaluddin y col 1996, Risco y col 1999). Los agentes infecciosos pueden ser responsables de disminución de las tasas de ovulación, fertilización, sobrevivencia embrionaria, sobrevivencia fetal y perinatal; sin embargo, algunos signos de enfermedades reproductivas pueden ser similares a anormalidades genéticas, efecto de toxinas o traumas físicos (Kirkbridge 1992, Gatica 1996).

Según sean la(s) etiología(s) o las combinaciones de ellas predominantes en un rebaño o región geográfica, la curva de sobrevivencia fetal va a tener patrones diferentes. Al analizar las curvas de sobrevivencia fetal -por ejemplo si se obtiene en la curva un peak en una etapa de la gestación- se puede sospechar de un mecanismo de aborto predominante; curvas bi o trimodales pueden indicar más de una etiología o la combinación de ellas (Thurmond y Picanso 1990). Además la curva de sobrevivencia fetal para vacas con diferentes características (edad, antecedentes de abortos anteriores) puede variar dentro de un mismo predio al mostrar una forma similar pero a distinto nivel (Thurmond y col 2005); no obstante, se necesitan datos de muy buena calidad para estimar estos efectos.

\section{IMPACTO PRODUCTIVO Y ECONOMICO DEL SAB}

Las pérdidas sobre la producción que produce el SAB deben identificarse integralmente, ya que no sólo corresponden a la potencial pérdida del ternero, sino también a todas las acciones que se debieron realizar para lograr la gestación en la vaca, como son gasto de semen, personal, alimentación, espacio ocupado en infraestructura, etc. Además se deben considerar las pérdidas productivas del peak de producción de leche futura no realizada como consecuencia del alargamiento del lapso entre partos, y de secuelas como infertilidad o de pérdidas embrionarias tempranas postaborto y alargamiento del intervalo generacional.

Los problemas de aborto pueden ser una importante causa de eliminación de vacas, pudiendo llegar al 30 o 40\% del total de reemplazos (Martin y col 1982), siendo más perjudicial cuando se aplica a animales que tengan vida útil o animales de gran valor genético dentro del rebaño. Se han realizado numerosos estudios, utilizando diferentes técnicas para estimar las pérdidas económicas producidas por los abortos en los sistemas productivos lecheros. Se reportan antecedentes para lecherías en California en la década de los 80 , donde se estimó que si una vaca aborta un feto de 100 días, significa una pérdida de US\$ 640 (Anonymous 1986, citado por Thurmond y Picanso 1990). No existen estimaciones de tal tipo para Chile.

\section{ANTECEDENTES DEL SAB EN CHILE}

En Chile, el Servicio Agrícola Ganadero (SAG) tiene implementado un sistema de atención de denuncias de enfermedades animales, el cual es un componente del
Sistema de Vigilancia Epidemiológica. En el período 1999-2004, la principal causa de denuncia en el SAG correspondió a la muerte de un animal $(48,1 \%)$, seguido del aborto, principalmente, en bovinos $(30,6 \%)$. Se debe considerar que la especie animal más denunciada es la bovina $(63 \%)$. Las enfermedades y patologías diagnosticadas por el SAG desde el año 1999 muestran que los diagnósticos de tipo infeccioso se agrupan en torno a enfermedades tales como diarrea viral bovina (DVB), brucelosis bovina y leptospirosis. Por ejemplo, en el año 2004, del total de denuncias realizadas de enfermedades y patologías infecciosas de la especie bovina, el 80,3\% correspondió a estas tres (Galleguillos y col 2005). Cabe destacar que DVB y brucelosis bovina son de notificación a la Organización Internacional de Epizootias (OIE 2007). Según datos de las denuncias en el SAG en el año 2001 en la Provincia de Valdivia, de los abortos observados analizados y con diagnóstico se determinó que un $50 \%$ fueron serológicamente positivos a herpes virus bovino tipo 1 (HVB), un 79,8\% a DVB, un $19,3 \%$ a leptospirosis, un $3,5 \%$ a brucelosis y un $62,2 \%$ a neosporosis, 10 que indica diagnósticos múltiples por caso. El $92 \%$ de los predios estaban insertos en el Programa del SAG de Certificación de Predios Libres y el 56,3\% estaba libre de brucelosis (Alacid 2001).

A nivel nacional existen estudios que investigan la presencia de agentes que son abortivos. En el caso de DVB se ha reportado $18 \%$ de bovinos portadores inmunotolerantes (Celedón y col 1998), una prevalencia regional en rebaños de 89 a 95\% (Meléndez y Donovan 2002), para la Décima Región de 69,4\% a 79,5\% (Reinhardt y col 2001, 2002). Respecto a otros agentes, se reporta el aislamiento de Mycoplasma bovis, desde el 7\% de muestras de tanques de lecherías analizados en predios de la zona sur (Sickles y col 2000). Además, según antecedentes aportados por Zamora y Riedemann (1999) en la zona sur de Chile existen serovares de leptospira pertenecientes a lo menos a L. interrogans y L. borgpeterseni y con una diseminación amplia en roedores silvestres y animales domésticos. Patitucci y col (1999) reportaron evidencia de que vacas con antecedentes de abortos presentaron anticuerpos contra N. caninum, con reportes de prevalencias prediales de 15,7 a 30,2\% (Patitucci y col 2000).

Del análisis de 70 informes anatomopatológicos realizados en el Instituto de Patología Animal de la Universidad Austral de Chile, de fetos bovinos provenientes de predios de la zona sur entre los años 2003 y 2005, como mayor causa de aborto diagnosticada se indican leptospirosis $(22,8 \%)$, brucelosis $(15,7 \%)$, lesiones compatibles con neosporosis $(10,0 \%)$ y DVB $(5,7 \%)$, además de $30 \%$ en que no se logra determinar la causa de aborto, lo que según los autores es principalmente debido a alteraciones postmortem (Paredes y Moroni 2005).

En general, las investigaciones que abordan la temática del aborto bovino a nivel nacional se enfocan al estudio de una patología en especial y no se analiza la presentación de estas enfermedades en relación a factores de rebaño o de 
los animales. Es necesario que los diseños de los estudios permitan realizar inferencias a la población general, o a estratos específicos de ellos; además es importante que permitan analizar los factores en forma conjunta e inferir relaciones causales más que de asociación estadística, para lo cual se precisan estudios prospectivos con adecuados grupos de comparación (Dohoo y col 2003).

\section{FACTORES DE RIESGO ASOCIADOS A LA PRESENTACION} DEL SAB

Asociados a la presentación de este síndrome hay factores atribuibles al animal, al ambiente y/o a agente (s) parasitarios e infecciosos presentes en el medio. Algunos estudios al analizar las etiologías de las pérdidas fetales se centran sólo en estos últimos (Alves 1996, Kirkbridge y col 1992, Otte y col 1995, Wolfgang 2003 ${ }^{\text {ab }}$ ), mientras que otros autores asocian estos elementos con otros componentes de la tríada epidemiológica, como son características de la vaca, antecedentes de las gestaciones anteriores, estación del año (Gröhn y col 1990, Thurmond y col $1990^{\mathrm{b}}$, Thurmond y Picanso 1993, Forar y col 1996, Jamaluddin y col 1996, Markusfeld 1996, Hovingh 2002, López-Gatius y col 2002), los cuales entregan una visión más amplia de los factores de riesgo que pueden estar participando en la ocurrencia de los abortos.

Factores intrínsecos de la vaca. En el período embrionario es cuando se produce el reconocimiento materno/fetal, el embrión produce señales que previenen la luteólisis, siendo el cuerpo lúteo necesario para la mantención de la preñez hasta alrededor del día 200 de la gestación. En los dos últimos meses la placenta debe ser capaz de mantener la gestación por la síntesis propia de progesterona y estrógenos (Stevenson 1997). Si por alguna razón la hembra recibiera una dosis adecuada de prostaglandina $\left(\mathrm{PGF}_{2}\right)$, sea endógena o exógena, se puede producir la pérdida de la gestación por luteólisis y contracción del miometrio. Se ha demostrado que un cuadro de mastitis o un cuadro febril aumentan el riesgo de abortar por esta vía (Risco y col 1999, Moore y col 2005).

La preñez con dos fetos también puede aumentar el riesgo de que la vaca aborte, lo cual ha sido documentado por López-Gatius y col (2002) quienes siguieron ecográficamente las preñeces en 601 vacas, de las cuales 10,6\% perdieron la gestación. Además, estimaron un riesgo 3,1 veces mayor de abortar para las vacas gestantes de dos fetos en relación a las con un feto. Sin embargo, no se especifica la edad de los fetos al ocurrir el aborto, lo que habría sido útil para caracterizar el aborto por este origen, de manera de poder ubicarlo como una causa temporal definida que aporte ideas frente a una curva de abortos específica de un rebaño.

Factores genéticos. Por lo general los reportes de abortos relacionados con problemas genéticos son muy escasos o poco documentados. Existen estudios que demuestran el aumento en la incidencia de pérdidas gestacionales en vacas inseminadas con semen de un toro en particular (sobre seis analizados) (López-Gatius y col 2002); sin embargo, no se hace mención a que dichos animales pertenezcan a una línea genética o familia en particular. Otras pérdidas gestacionales se pueden asociar a defectos genéticos propios del embrión, como, por ejemplo, deficiencia de la enzima uridina monofosfato sintetasa (Moore y col 2005).

Edad de la vaca. Se reporta que el riesgo de aborto de las vaquillas es menor que el de las vacas de segundo parto (Markusfeld 1996) y que el riesgo de aborto también es mayor en vacas de más de 5 años (Thurmond y col $\left.1990^{\mathrm{a}}\right)$.

Sin embargo, hay autores que no han encontrado asociación significativa de la edad de la vaca o del número de lactancias con la presentación de abortos (Dohoo y Martin 1984, Gröhn y col 1990, Thurmond y col 1990 , López-Gatius y col 2002).

Se ha reportado que las vacas de menos partos necesitan un período de descanso más largo para recuperarse del stress postparto y cubrir el incremento en la demanda nutricional de crecimiento y producción de leche durante los primeros años (Sanz 2004).

Producción de leche. El efecto de la producción de leche en la lactancia anterior o en curso en relación a los problemas reproductivos ha sido evaluado en varios estudios, los que señalan resultados contradictorios y no son uniformes los problemas reproductivos analizados. Es así como Erb y col $\left(1981^{\mathrm{ab}}\right)$ reportaron que la producción de leche tiene poco efecto en los problemas reproductivos; posteriormente Erb y col (1985) y Bigras-Poulin y col (1990) encontraron asociación entre vacas de alta producción de leche y la presentación de ovarios quísticos, pero no hacen mención a la presentación de abortos y la producción láctea. Gröhn y col (1990) tomaron en cuenta la presentación de abortos y el nivel de producción y concluyeron que el incremento en la producción individual de una vaca respecto a la lactancia anterior aumenta su riesgo de aborto, retención de placenta, metritis y celo silente.

Influencia de patologías y abortos anteriores. Las enfermedades infecciosas y parasitarias son el foco primario en las medidas de prevención de los abortos; sin embargo, estos agentes probablemente causen menos de la mitad del total de las muertes fetales (Barr y Anderson 1993).

La brucelosis es una enfermedad zoonótica causada por bacterias del género Brucella, siendo la especie específica para el bovino Brucella abortus. La fuente de infección son los fetos abortados, descarga uterina, placenta y leche; el rebaño afectado presenta abortos, momificación de fetos o retención de ellos, nacimiento de terneros débiles o muertos, retención de placenta e infertilidad mayor a los niveles normales. Se estima que se puede llegar a $80 \%$ de 
abortos en vacas no vacunadas e infectadas en el primer trimestre de gestación. Los abortos ocurren normalmente de los 6 a 9 meses. El diagnóstico se puede realizar mediante el aislamiento de la bacteria de fluido uterino, leche, placenta, pulmón del feto, contenido estomacal del feto, o por técnicas de aglutinación serológicas o en la leche. El estado portador de la vaca puede llegar a ser permanente (Miller 1986).

La leptospirosis también es una enfermedad zoonótica causada por varios serovares, y el mantenido por el bovino es el serovar hardjo (Ellis 1994); los reservorios principales del agente son animales domésticos infectados, animales silvestres, además de agua o alimento contaminado con orina de animales infectados. La tasa de aborto en predios infectados puede ser muy variable, de 5 a $40 \%$ en forma esporádica o epidémica, usualmente en el último trimestre de la gestación (Miller 1986, Ellis 1994).

Listeriosis es una enfermedad infecciosa zoonótica y transmitida por los alimentos, causada por Listeria monocitogenes. Son portadores una gran cantidad de mamíferos, aves y peces que excretan el microorganismo en las fecas, el cual sobrevive en fecas secas hasta por dos años, constituyéndose en una infección de tipo ambiental ubicua; sobrevive especialmente bien en el ensilaje, por lo que éste es una muy buena fuente de transmisión (Miller 1986). Hassan y col (2000) encontraron un aumento en la prevalencia predial en primavera. Se establece una infección latente y frente a una condición de estrés resulta en placentitis y septicemia fetal. En la hembra puede haber pirexia y retención de placenta. En el rebaño el aborto puede ser esporádico o múltiple hasta $50 \%$, ocurriendo la mayoría en el último trimestre, pudiendo recurrir (Miller 1986).

Dentro de las enfermedades parasitarias la neosporosis es importante a nivel mundial (Alves y col 1996); este protozoario es transmitido por el perro mediante huevos $\mathrm{u}$ ooquistes en la materia fecal que al ser consumida por el bovino producen la infección (Anderson 1994, Paré y col 1997); además se ha comprobado que tanto la seroprevalencia del rebaño como la probabilidad de transmisión horizontal son mayores en granjas que tienen perros (Bartels y col 2007). La mayoría de los abortos por esta causa se observan durante los cuatro a seis meses de preñez, siendo común que el feto presente autólisis; si el ternero llega a nacer se observaron problemas neurológicos. Los abortos se pueden presentar como tormentas o en forma continua en el rebaño, según sea el tipo de infección que exista en el predio. Las vacas seropositivas tienen dos a tres veces más probabilidad de abortar que las seronegativas; los animales infectados conservan su condición de portadores permanentemente y la descendencia continuará siendo seropositiva e infectada, disminuyendo la producción de leche y con mayor predisposición a otras infecciones (Wouda y col 1998). Se ha demostrado que la alta prevalencia y la ocurrencia de brotes se relacionan con la presentación de abortos, eliminación prematura de las vacas y disminución en la producción de leche (Bartels y col 2006).
El virus de la diarrea viral bovina tiene un importante impacto en la salud reproductiva del rebaño. La infección de la hembra durante el período de preimplantación (de 30 a 40 días) puede resultar en muerte embrionaria, mientras que infecciones entre los 40 y 125 días se caracterizan por muerte fetal, aborto, momificación y nacimiento de terneros permanentemente infectados e inmunotolerantes (Moennig y Liess 1995). La infección posterior a los 125 días puede resultar en defectos congénitos. El ternero, aparentemente sano pero resultante de una infección congénita sufre un impacto negativo en su salud posterior, lo que finalmente impacta la salud del rebaño (Muñoz-Zanzi y col 2003). El contacto directo con animales permanentemente infectados es con probabilidad la vía de transmisión más importante (Houe 1995). La tasa de aborto es usualmente baja, los abortos ocurren tempranamente, pero por lo general sobre los cuatro meses (Miller 1986).

En la infección con el virus de la rinotraqueítis infecciosa bovina el virus se puede alojar en la placenta o en el feto y provocar su muerte en 24 horas. En la vaca el aborto puede ser posterior a la forma respiratoria o conjuntival de la enfermedad, pero también puede que no se presenten signos. El aborto por esta causa puede variar entre 5 a $60 \%$ de las vacas infectadas y puede ocurrir entre los cuatro meses al término de la gestación. El estado de portador de los infectados puede ser permanente (Miller 1986).

Los agentes infecciosos pueden actuar por sí solos, pero también pueden hacerlo en forma conjunta u oportunista frente a la coinfección con algún otro agente. Para las enfermedades abortigénicas se tiene evidencia de que por ejemplo Campylobacter foetus, Brucella abortus, el virus DVB, además de poder producir trastornos reproductivos por sí mismos producen cambios en la placenta que permiten a otros patógenos cruzar la barrera placento-fetal más fácilmente (McGowan y Kirkland 1995). Es común observar asociaciones entre la presencia de anticuerpos contra Neospora caninum y DVB en las vacas que han abortado, y es muy importante tener en cuenta el potencial inmunosupresivo de la DVB, el cual incrementaría la susceptibilidad y severidad de los signos clínicos de otros agentes infecciosos (Muñoz-Zanzi y col 2003). Además de las infecciones mixtas en los órganos reproductivos, puede haber infecciones en otros órganos que afecten el ciclo reproductivo, ya sea alterando el intervalo entre estros o causando abortos. Una situación interesante es el efecto que pueden tener en el útero mediadores de la inflamación, como las prostaglandinas producidas por endotoxinas de las bacterias gram-negativas y la respuesta inflamatoria de las bacterias gram-positivas, las que pueden llegar a producir aborto (Giri y col 1990, citado por Risco y col 1999). Otros estudios estimaron que el riesgo de aborto en vacas que cursan con mastitis durante los primeros 45 días de gestación es 2,7 veces mayor que el riesgo de aborto durante los próximos 90 días que las vacas sin mastitis clínica (Risco y col 1999, Wolfgang 2003 a).

Miller (1986) señala que si la causa predominante de aborto en una vaca fuese de origen infeccioso (tricomo- 
niasis, neosporosis, listeriosis, brucelosis, y varias cepas de leptospirosis) se esperaría una repetición de aborto, postura cuestionable si se considera la inmunidad que se pudiese generar (Ellis 1994, Paré y col 1997). Al analizar la recurrencia de abortos en dos lactancias, Dohoo y Martin (1984) no encontraron asociación entre el aborto que ocurrió en una lactancia y el riesgo de abortar en la siguiente. Los mismos autores evaluaron la relación de las enfermedades dentro de la misma lactancia en base a un análisis de vía; el aborto se asoció significativamente con enfermedades de los miembros o pezuñas y con la presentación de fiebre de leche. Estas asociaciones pueden ser evidencia de una causa potencial no infecciosa, pero requiere de mayor análisis. Cabe mencionar que los indicadores de riesgo en dicho estudio pudieron tener sesgo debido al bajo número de observaciones en algunas asociaciones, además en los modelos no se controló por la edad, la que frecuentemente es factor de confusión. Posterior al aborto pueden presentarse consecuencias en el rendimiento reproductivo de la vaca; es así como Gröhn y col (1990) estimaron que si una vaca aborta, tiene 19,8 veces más probabilidad de tener retención de placenta, 3,7 más probabilidad de tener metritis temprana y 2,1 más probabilidad de tener un celo silente. De manera inversa, aumenta la probabilidad de abortar el que una vaca tenga retención de placenta o metritis temprana en 11,4 y 2,3 veces, respectivamente. Se debe apreciar que este análisis es el resumen de 10 modelos de regresión logística, uno para cada desorden reproductivo, por lo que se pierde la posibilidad de ajustar la posible correlación entre las diferentes variables dependientes en su asociación con las variables dependientes.

Thurmond y col $\left(1990^{a}\right)$ señalan que la proporción de abortos para vacas con historial de abortos fue de $14,5 \%$, mientras que para las vacas sin antecedentes de abortos fue de $12,1 \%$. Siguiendo la misma línea, Thurmond y col (2005) muestran diferencias en las curvas de sobrevivencia fetal para vacas que ya tuvieron un aborto en relación a las que han tenido dos abortos. En dicho estudio todos los grupos presentaron una distribución de abortos trimodal, lo que sugiere que estuviera presente la(s) misma(s) etiología(s) en vacas con uno o dos abortos anteriores. El efecto fue mayor si la vaca tuvo dos eventos anteriores y si ellos ocurrieron tardíamente en la gestación (Thurmond y col 2005), lo cual podría ser efecto de una enfermedad infecciosa como neospora (Bartlet y col 2006, Pabón y col 2007).

Factores relacionados con la alimentación y el ambiente. El potencial efecto de las plantas tóxicas pudiera presentarse con mayor frecuencia en sistemas de alimentación en que el animal no puede discriminar el alimento que recibe, por ejemplo en forma de soiling en un patio de alimentación. En cambio, en condiciones en las cuales el animal puede efectuar la selección en el consumo él podría evitar la ingesta de plantas de mal sabor o muy groseras, ya que algunas plantas tóxicas poseen baja palatabilidad.
El cambio en la utilización de alimentos de acuerdo a la disponibilidad puede influir en cuanto al rol de las micotoxicosis, intoxicaciones con nitratos (Miller 1986) o algunas enfermedades bacterianas como listeriosis relacionada con la utilización de ensilaje (Hassan y col 2000).

Los rumiantes son especialmente sensibles a intoxicaciones por nitritos, resultando en un estrés hipóxico, lo que puede producir el aborto por hipoxemia fetal; también, según la edad del feto, pueden activarse las glándulas adrenales, con el consecuente aumento del cortisol fetal, lo que puede producir un aborto tardío en vacas con exposición a altas dosis (Casteel 1997). Se pueden presentar problemas de intoxicaciones alimentarias por micotoxinas que produzcan abortos, como es el caso de la festuca parasitada con hongos, alcaloides tipo ergotamina producidos por hongos del género Claviceps o aflatoxinas en los granos; cabe destacar que algunas micotoxinas, en especial las aflatoxinas y tricotecenos, tienen un importante efecto inmunosupresor, que puede potenciar la presentación de abortos de tipo infeccioso (Casteel 1997). En cuanto a factores relacionados con la nutrición que afecten la presentación de abortos en forma temprana, Moore y col (2005) señalan que una disminución en la condición corporal puede aumentar las pérdidas entre los 40 a 90 días de la gestación debido a una influencia en la producción de progesterona por el cuerpo lúteo. Las deficiencias de minerales y vitaminas pueden ser un importante factor para la presentación de abortos, especialmente en el período final de la gestación, por lo que se confunden entre abortos tardíos y nacimientos muertos.

Existen antecedentes del cambio del riesgo de aborto durante el año. Algunas de las posibles fuentes de variación pueden ser los cambios estacionales de la función endocrina, que mejora con el aumento de las horas de luz (Hansen 1997) o la presencia de vectores que puedan variar durante el año (Miller 1986). Barr y col (1990) reportan que los casos de aborto remitidos al sistema de diagnóstico veterinario de California aumentan en los meses de otoño e invierno. Está documentado que las condiciones de calor y humedad afectan la tasa de concepción (García-Ispierto y col 2007) y que la tasa de abortos es más alta en vacas que conciben en la estación calurosa (López-Gatius y col 2004).

Factores relacionados con el manejo. Se ha reportado como una práctica negativa el inseminar una vaca que está preñada (Sturman y col 2000). La pérdida de gestación al reinseminar un grupo de vacas preñadas fue de $24 \%$, significativamente mayor $(\mathrm{P}<0,05)$ que el $7 \%$ de pérdidas en las vacas no reinseminadas. También pueden ser causales de abortos tardíos los manejos poco cuidadosos y bruscos de vacas en gestación avanzada (Miller 1986), los que pueden ser favorecidos por condiciones como alta densidad en patios de alimentación o en los patios de espera, caídas por superficies muy lisas o procedimientos en la manga a vacas con más de siete meses de gestación que puedan producir caídas o compresiones fuertes del útero. 
Existe relativamente poca documentación respecto a la influencia de los sistemas de producción en la presentación de abortos. Dentro de los potenciales factores de riesgo es importante destacar la posible relación, por ejemplo, entre tipo de estabulación de los animales; la estabulación grupal (sin separación individual de los animales) se asocia a un mayor error en la detección de estros que el de estabulación individual (Reimers y col 1985), lo que conlleva a que si hay un alto porcentaje de error en la detección de estros pueden estarse inseminando vacas gestantes, lo cual influye en un mayor riesgo de abortos (Sturman y col 2000). También es dependiente del sistema de producción la separación que existe entre las diferentes categorías de bovinos, los sistemas de pastoreo, el origen del agua de bebida, el acceso a fuentes de aguas estancadas y el control de vectores que se realiza en el predio. La mayoría de los estudios que buscan asociaciones de factores multicausales con el aborto bovino han sido realizados en Norteamérica (Thurmond y col 1990a , Thurmond y Picanso 1990, Lemire y col 1993, Otte y col 1995, Alves y col 1996, Forar y col 1996, Markusfeld 1996, López-Gatius y col 2002, Hanson y col 2003, Thurmond y col 2005) y no hacen referencia directa a alguna clasificación del sistema de producción que poseen las lecherías estudiadas, probablemente por ser muy similares entre ellas.

\section{IDENTIFICACION DE LA ETIOLOGIA DE LOS ABORTOS}

La identificación de la causa de un problema de aborto a nivel predial es usualmente un desafío tanto para el veterinario clínico como para el laboratorista. El sentido de conocer la causa de los abortos es lograr su prevención. Para lograr conocer las causas, las herramientas epidemiológicas contribuyen significativamente en orientar la investigación, de manera de lograr una mejor interpretación de los resultados de las pruebas diagnósticas y de integrar la información. Sin embargo, en ocasiones no se logra encontrar una causa definida debido a razones de imperfecciones de los test, etiologías no infecciosas, etc. Las posibilidades de éxito se podrían incrementar significativamente si se articula una investigación epidemiológica que involucre el rebaño, conjuntamente con un muestreo sistemático y preciso, además de una acertada interpretación de los resultados aportados por las pruebas diagnósticas (Thurmond y col 1990ª). Pese a que este aspecto se conoce, es común que al estudiar el aborto desde el comienzo del análisis sólo se busquen etiologías infecciosas (Kirkbridge y col 1992, Alacid 2001, Campero 2003, Wolfgang 2003 ${ }^{\mathrm{ab}}$ ) sin tomar en cuenta los demás factores que inciden en el síndrome.

Otro importante principio es que para que exista asociación causal, la exposición a la causa debe ocurrir antes que se observe el efecto (Thrusfield 1995). Frecuentemente esta situación no se puede comprobar con las muestras de campo. A menudo el aborto es el resultado de un incidente (infeccioso o no infeccioso) que pudo ocurrir semanas o meses antes de que el aborto se diagnostique o que se observe. La tasa de observación de los abortos varía entre 2 a 5\% (Kirkbridge y col 1992) o del 20\% (Forar y col 1996). Además se logra diagnosticar en alrededor del $50 \%$ de los casos la causa de los abortos observados, situación que se reporta tanto a nivel nacional (Paredes y Moroni 2005) como internacional (Alves y col 1996, Campero 2003, Givens 2006).

Si bien el diagnóstico etiológico es una información importante, el SAB es un problema de rebaños. Es por eso que, aunque la causa específica no se pueda detectar al momento en que se produce el aborto, éste sea una secuela del verdadero evento que lo produjo (Thurmond y col 1990a , Kirkbridge 1990, 1992); para llegar a un diagnóstico se debe realizar una investigación epidemiológica que incorpore una serie de evidencias. Por ejemplo, se debe tomar en cuenta la distribución temporal de los casos de aborto y abarcar todos los posibles factores de riesgo, ya sean propios de la vaca (antecedentes de abortos, gestación de un solo feto o mellizos, nivel de producción, mastitis), del ambiente (tipos de manejo, tipo y cambios de alimentación, antecedentes de bioseguridad, etc.) y evaluar los antecedentes que hagan sospechar de enfermedades infecciosas o parasitarias (signología clínica, estado inmunológico aportado por vacunas, características del feto, de las membranas fetales y de las condiciones de la vaca).

Los datos recolectados rutinariamente de los laboratorios de diagnóstico veterinarios no son tasas verdaderas sino tasas proporcionales, que si bien pueden ser útiles para el estudio de una enfermedad generalmente carecen de la información adecuada de la población en riesgo de la que provienen. Por esto no son necesariamente una estimación confiable de la frecuencia de la enfermedad en la población. Además un diagnóstico etiológico certero del aborto en el laboratorio depende, entre otras cosas, de la disponibilidad de una buena historia clínica, una adecuada recolección, conservación y envío de las muestras (Kirkbridge 1990, Rivera 2001 Paredes y Moroni 2005). Jamaluddin y col (1996) entregan evidencia de que cuando se remite al laboratorio el feto, placenta, sangre materna o sus combinaciones en estado fresco y no autolítico es más probable obtener una alta tasa de diagnóstico definitivo. Por otra parte, hay factores asociados al laboratorio, como son nivel y disponibilidad de las capacidades diagnósticas y cuán involucrado esté a programas de monitoreo y vigilancia de abortos, lo que puede influir privilegiando ciertas etiologías.

Un factor importante que puede generar sesgo es que frecuentemente los envíos del material biológico de los abortos a los laboratorios se originan de clientes habituales de ellos, los que pueden ser no representativos de la población general de animales que han tenido abortos (Thurmond y col 1994, Jamaluddin y col 1996).

La interpretación de los resultados de una prueba diagnóstica debe realizarse teniendo en cuenta la historia clínica del animal, del rebaño, medio ambiente, etc., to- 
mando el contexto predial. Los resultados serológicos en casos de abortos deben analizarse con sumo cuidado, pues éstos dependerán de la prevalencia de la infección en una determinada área y de la persistencia de los anticuerpos en el animal (Wolfgang 2003 ${ }^{\mathrm{ab}}$ ), como también de la sensibilidad y especificidad de la prueba.

Por ejemplo, en el caso de DVB, el aborto puede aparecer en cualquier momento de la gestación y no está necesariamente asociado con el momento en que se infectó la vaca, por lo que constatar o no la seroconversión no permite asegurar ni desechar una hipótesis causal (Lindberg 2002). En caso de infección bacteriana, tiene importancia el aislamiento puro de un determinado agente, de lo contrario puede tratarse de contaminaciones postaborto.

El utilizar vacunas como método preventivo para algunas enfermedades infecciosas que producen aborto puede controlar los brotes epidémicos de abortos, pero presenta el inconveniente de que limita la posibilidad de utilizar las pruebas diagnósticas serológicas, que son las que más frecuentemente se utilizan en la búsqueda de las posibles causas del aborto a nivel predial. Esto se debe a que la mayoría de las pruebas diagnósticas actualmente disponibles están basadas en reacciones inmunológicas y no distinguen entre anticuerpos generados tras una infección de aquellos producidos tras una vacunación (Hovingh 2002). Además, la seroconversión de anticuerpos séricos en la madre o aún el aislamiento del agente en el feto no necesariamente es prueba suficiente de que un determinado agente infeccioso fue la causa del aborto (Thurmond y Picanso $1990^{\mathrm{b}}$ ), ya que, por ejemplo, un feto puede no estar infectado y factores que afecten a la madre provoquen la interrupción de la gestación, concomitancia de varios agentes, etc.

\section{PREVENCION DEL SINDROME DE ABORTO BOVINO}

Las prácticas de bioseguridad, es decir, las medidas que minimizan el riesgo de introducir una enfermedad al predio y prevenir su diseminación, pueden incluir cuarentena de los animales, mantenimiento del rebaño en forma cerrada, restricción de las visitas en cuanto a evitar la transmisión indirecta de agentes. Estas son medidas que contribuyen a la salud del rebaño en general e incluyen la prevención de la introducción y diseminación de enfermedades abortigénicas. Por otra parte, el mantener un ambiente limpio y confortable para los animales, una adecuada alimentación que satisfaga sus requerimientos, contribuyen a minimizar el estrés, que puede ser un factor predisponente para desarrollar alguna de las enfermedades que producen pérdidas fetales (Hovingh 2002).

Si bien la práctica de vacunar tiende principalmente a mitigar los efectos de los brotes de aborto clínicos, el vacunar esporádicamente contra un grupo variado de posibles agentes generalmente no controla definitivamente el problema; en muchas situaciones posterga el control e incrementa sus costos. Adicionalmente, también es frecuente que los animales estén coinfectados por dos o más agentes, por lo que al tomar una decisión sobre qué vacunas administrar se deben considerar los antecedentes epidemiológicos de las enfermedades presentes en el rebaño y en la zona, además de las medidas de manejo propias del rebaño (Hovingh 2002).

Es sumamente importante para el sector ganadero nacional contar con un enfoque racional y relativamente de fácil implementación que permita abordar el control de esta importante problemática de manera clara y precisa, de manera tal de poder incrementar las posibilidades de conocer las causas de los problemas abortivos a nivel predial y/o guiar al profesional o productor en el control efectivo de las causas que los ocasionan, monitorear los alcances de las medidas implementadas y no sólo disminuir los efectos del problema. Por ejemplo, la habilidad de predecir qué vacas tienen más probabilidad de abortar podría promover la salud del rebaño al mejorar las decisiones sobre aquellas vacas que deberían ser retenidas. Conjuntamente, el conocimiento de qué períodos de riesgo son los más probables en un establecimiento determinado (por ejemplo temprano o tardío en la gestación, estación del año, etc.) podría ser útil en guiar el tipo de pruebas diagnósticas a solicitar para obtener evidencias de exposición previa al período de riesgo a toxinas o agentes infecciosos. Otra importante ventaja de este enfoque es que el uso de una metodología estandarizada permite la comparación de riesgos en el tiempo, entre predios de una misma zona, o de otras regiones, lo que facilita no sólo el monitoreo a nivel predial sino también de toda la cadena de producción primaria en Chile.

\section{PARTE 2}

\section{ASPECTOS METODOLOGICOS EN EL ESTUDIO DEL SAB}

Las metodologías más utilizadas para cuantificar los abortos en los estudios epidemiológicos que tratan el tema son una caracterización mediante el uso de proporción de abortos y tasas de densidad de abortos brutas y específicas, mientras que para identificar los períodos de mayor riesgo y describir la función de riesgo de aborto se utiliza el análisis de sobrevivencia. Se han hecho modificaciones a este método para considerar abortos que se repiten de un período a otro.

\section{EVENTOS NECESARIOS PARA EL CALCULO DE INDICADORES}

Para explicar claramente los indicadores de aborto utilizados en una investigación es necesario tener definidos lo más precisamente posible los siguientes aspectos (adaptado de Thurmond y Picanso 1990): definición de caso de aborto, período en riesgo de abortar, técnica utilizada para diagnosticar preñez y estado de la vaca en cada revisión de control, identificación de las vacas y 
fecha en que se ha realizado el servicio o inseminaciones artificiales y sus repeticiones, identificación de las vacas que se han diagnosticado preñadas, fecha del parto anterior, fecha del término del período en riesgo o de seguimiento de cada vaca.

Es necesario recurrir a la inspección e interpretación de los registros reproductivos para poder detectar los abortos no observados y con esto asegurar que el numerador de los indicadores epidemiológicos de los abortos sea reflejo de la realidad del rebaño. Resulta de utilidad realizar un análisis de intervalos entre repeticiones de las inseminaciones artificiales o celos, ya que podría haber habido una muerte fetal si el celo o inseminación se repite a más de 42 días después de la última inseminación/celo, ya que las repeticiones cercanas a los 21 días pueden corresponder a que no hubo concepción u ocurrió una muerte embrionaria antes del período de reconocimiento materno (Stevenson 1997). Repeticiones de entre 21 y 42 días corresponderían a mortalidades embrionarias y no fetales, esto siempre que la detección de celos sea perfecta. El lapso interparto menor a 260 días o lactancias cortas, es decir, de menos de 8 meses, pueden ser indicadores de que la vaca se secó antes de lo debido, entre otras causas, por haber cesado la gestación. Es importante también la revisión del estado de gestación de las vacas que ya se han diagnosticado como preñadas y que presenten celo, ya que la presentación de comportamiento de celo puede ser normal en aproximadamente 5\% de las vacas gestantes (Donald 1943, citado por Sturman 2000).

\section{MEDIDAS DE FRECUENCIA PARA CUANTIFICACION DE SAB}

En el diseño de los estudios que cuantifiquen este síndrome o identifiquen factores de riesgo se deben establecer claramente la población en riesgo, las definiciones de los eventos y los procedimientos analíticos a utilizar, ya que las principales inconsistencias al momento de realizar comparaciones entre los estudios están dadas por estas imprecisiones. Además debe tener claridad en los posibles sesgos asociados que puedan existir, por ejemplo a la presión de selección que se ejerce en los rebaños al eliminar las vacas que hayan abortado (Thurmond y col 1990a, Forar y col 1995, Forar y col 1996). Por otra parte, los estudios deberían tener en cuenta que el riesgo de aborto es mayor en la gestación temprana (Forar y col 1995, 1996) y que sólo considerar aquellos abortos que se detectan visualmente subestima la cantidad real que se producen.

Esta situación se reporta muy claramente en el amplio rango de observaciones de pérdidas fetales reportadas en la literatura, según lo demuestra una recopilación realizada por Forar y col (1995) en base a 26 estudios, donde el rango de pérdidas fetales se extendía del 0,4 al 10,6\%; sin embargo, al separar los trabajos entre los que registraban las pérdidas fetales sólo mediante la observación de ellas, el rango se redujo a $0,4-5,5 \%$, con una media de $2,3 \%$, mientras que en los que se registraban las pérdidas fetales de varias formas y no sólo mediante la observación directa, el rango fue de 3,6-10,6\% con una media de $6,8 \%$. Los autores coinciden en que se puede considerar como endémica una presentación de abortos de menos del 10\%, pero si se sobrepasa este nivel se puede estar en presencia de un brote o tormenta de abortos (Fetrow y col 1990). Esta información debe tomarse con cautela, en cuanto a que los niveles "normales" pueden ser muy variables dependiendo de los sistemas productivos, región geográfica, características de la raza, sistemas de manejo y enfermedades endémicas que sean abortivas. Sin embargo, otros autores consideran como normal que aproximadamente el 3 a $4 \%$ de las vacas identificadas como preñadas entre los 30 a 50 días podrían abortar o parir un ternero muerto. Del 4 a $6 \%$ de las preñeces pueden terminan en un parto con ternero muerto y de éstos entre un 7 y $28 \%$ pueden poseer anormalidades congénitas, $84 \%$ puede morir debido a asfixia neonatal y $9,1 \%$ puede tener evidencia de una infección (Miller 1986).

La epidemiología descriptiva proporciona herramientas útiles para definir y cuantificar una situación y entender la naturaleza de un problema. Los indicadores más utilizados para cuantificar los abortos en un rebaño son la proporción de abortos, la incidencia de abortos y para su análisis cuantitativo el análisis de sobrevivencia.

Proporción de abortos (PA). Es el indicador que requiere de información más general y es al que se recurre más frecuentemente. Este debiera calcularse considerando en el numerador los abortos ocurridos y en el denominador, el total de gestaciones en un período determinado, por ejemplo durante un año. Puede utilizarse en forma cruda (en base a todas las hembras) o estratificarla según edad, raza, nivel de producción, etc. (Thrusfield 1995). Esta medida si bien es objetiva y entrega información acerca del subgrupo de animales en que se concentra el problema, no dice nada acerca de su dinámica. Puede ser de mucha utilidad para caracterizar de forma general el rebaño o hacer evaluaciones después de alguna intervención, sin embargo no es la herramienta apropiada para detectar asociaciones causales en rebaños problemas. Este indicador no tiene sesgo respecto a la distribución de riesgo en la gestación, puede determinarse para una cohorte o en una tabla de vida contemporánea, donde se cuantifica para cada intervalo del período de riesgo. La PA debe calcularse de acuerdo a la probabilidad condicional de que una vaca no aborte antes de los 260 días de gestación (Thurmond y Picanso 1990).

Tasas de incidencia de aborto (TIA). Es el indicador de morbilidad más apropiado para caracterizar la dinámica de la ocurrencia de un evento, ya que indica el número de nuevos eventos en una población y períodos definidos. En el numerador se contabilizan los casos nuevos de aborto que ocurren en un período determinado, dentro de un grupo de animales que están en riesgo de que ello 
les ocurra. La tasa de incidencia puede tener sesgo en cuanto a la representación de riesgo en períodos que estén sobrerrepresentados por vacas en estados de gestación no asociados normalmente con algún riesgo de aborto (Thurmond y Picanso 1990).

\section{USO DE MODELOS PARA ESTUDIAR EL SINDROME DE ABORTO BOVINO}

La elección del modelo estadístico para estudiar una enfermedad tiene directa relación con los supuestos que se deben cumplir en ellos de acuerdo a la biología del problema. Los resultados de un análisis estadístico son función de los datos y supuestos utilizados, es por ello que es necesario analizar si los supuestos del modelo elegido tienen sentido con la verdadera estructura poblacional de la variable estudiada, ya que si asume diferentes supuestos sobre los datos los resultados serán diferentes (Greenland y Robins 1986, Maldonado y Greenland 1993). La distribución del tiempo de ocurrencia de aborto no es simétrica y comúnmente es bi o trimodal (Hanson y col 2003), por lo que no es apropiado analizarla con regresión lineal (Cleves 2004).

Los modelos de sobrevivencia son apropiados para analizar este tipo de datos (Dohoo y col 2003), los cuales permiten caracterizar los eventos de aborto en función del momento en el tiempo en que ocurre, mediante la estimación de la función de sobrevivencia fetal y la función de riesgo de morir de un feto. Es una herramienta muy útil para estudiar la asociación entre diferentes factores y el riesgo de que ocurra un evento, en este caso aborto, ajustando los diferentes tiempos en riesgo en los que los diferentes animales contribuyen durante el tiempo de observación (Dohoo y col 2003).

Los modelos de regresión logística se pueden utilizar para analizar la probabilidad de que una vaca aborte. Es posible conformar un modelo multivariable para analizar de forma conjunta el efecto de diferentes factores (Dohoo y col 2003).

Los indicadores de los modelos de sobrevivencia y de regresión logística se pueden interpretar como indicadores de riesgo (Dohoo y col 2003); sin embargo, si se dispone de la información del momento del aborto, los modelos de sobrevivencia ocupan un denominador más ajustado en los indicadores de riesgo (Kleinbaum 1996).

\section{SISTEMAS DE VIGILANCIA DE ABORTOS EN BOVINO}

Dada la posible falta de relación temporal entre el aborto y la causa que lo produjo, es difícil obtener datos precisos de la ocurrencia de abortos para encontrar una asociación causal. Esto lleva a que sea conveniente intentar mantener registros prediales de los posibles factores de riesgo de aborto, de manera de poder realizar un análisis retrospectivo predial acucioso en el momento que se presente un aborto.
Para implementar un sistema de vigilancia efectivo de una enfermedad se necesita a lo menos: tener un adecuado método de monitoreo que permita detectar rápidamente los eventos de interés, definir y evaluar los resultados en relación con el tiempo, establecer un criterio que permita concluir que los indicadores obtenidos en un rebaño o zona son mayores a los esperados. Es usual que el monitoreo del riesgo de aborto en un período calendario se realice mediante PA y/o TIA (Thurmond y Picanso 1990). Una alternativa tradicional es establecer la Curva Endémica y el Índice Endémico de la presentación de abortos con datos retrospectivos.

El inconveniente es que estos sistemas no incluyen la cuantificación de los costos asociados con la correcta o incorrecta identificación de un estado temprano de una epidemia. Los criterios para desarrollar un sistema de vigilancia de enfermedades óptimo dependen de las características de ellas, los costos de la enfermedad y los de su control, la probabilidad de que ocurra una epidemia y la magnitud esperada para seleccionar el número de agrupación de casos necesarios para activar la alarma. Por ejemplo, para enfermedades raras, el énfasis debería estar en evitar una falsa alarma más que en detectar si la epidemia ha ocurrido, en cambio, para enfermedades más comunes lo óptimo será un sistema de detección más sensible (Carpenter 2001).

\section{CONCLUSIONES}

El problema del síndrome de aborto bovino es un desafío para la Medicina Veterinaria, ya que implica pérdidas económicas para los ganaderos y es reflejo de medidas de manejo inadecuadas y malas condiciones sanitarias.

Existe mucha disparidad en los estudios que tratan el tema de aborto bovino, en la presentación y formas de cálculo de indicadores epidemiológicos para el análisis de la presentación y factores de riesgo, así como en las definiciones empleadas para identificar los eventos de importancia. Hacen falta estudios de cohorte para el análisis de factores de riesgo específicos y así poder evaluar su importancia relativa frente a otros.

Es necesario hacer una diferenciación clara entre los factores de riesgo del animal y del rebaño, lo que puede estar asociado a prácticas de manejo específicas que dependen del tipo de sistema productivo adoptado.

En Chile se necesitan estudios que consideren el problema de síndrome aborto bovino en forma integral. Además, los factores de riesgo establecidos en otros países no son totalmente extrapolables a las condiciones nacionales, por lo que se hace necesaria una mayor investigación en esta área para realizar un diagnóstico ajustado a la realidad nacional de manera de plantear sistemas de intervención eficientes y efectivos. 


\section{RESUMEN}

El aborto bovino es un factor limitante en las lecherías, por su impacto en la disminución de los reemplazos y producción del rebaño, por lo que constituye un desafío para la Medicina Veterinaria. El objetivo de este artículo es revisar los principales aspectos relacionados con la epidemiología y formas de análisis del Síndrome de Aborto Bovino (SAB). El trabajo se organiza en dos partes, la primera incluye aspectos epidemiológicos, impacto y factores de riesgo; en la segunda se presentan algunas metodologías para su cuantificación, análisis y sistemas de vigilancia. En los estudios que tratan el tema de aborto bovino existe mucha disparidad en la presentación y formas de cálculo de indicadores epidemiológicos, así como en las definiciones empleadas para definir los eventos de importancia que se utilizan para el análisis de la presentación y factores de riesgo. Es necesario hacer una diferenciación clara entre los factores de riesgo del animal y del rebaño, los que pueden estar asociados a prácticas de manejo específicas que dependen del tipo de sistema productivo adoptado. En Chile se necesitan estudios que consideren el problema del $\mathrm{SAB}$ en forma integral. Además, los factores de riesgo establecidos en otros países no son totalmente extrapolables a las condiciones nacionales por lo que se hace necesaria una mayor investigación en esta área para realizar un estudio ajustado a la realidad nacional que permita diseñar sistemas de intervención y control eficientes y efectivos.

\section{AGRADECIMIENTOS}

A la Universidad de Concepción y CONICYT por permitir la realización del Programa de Doctorado en Ciencias Veterinarias.

\section{REFERENCIAS}

Alacid M. 2001. Descripción epidemiológica del Síndrome Abortivo Bovino en rebaños sometidos a monitoreo por el Servicio Agrícola y Ganadero en ocho comunas de la Provincia de Valdivia, período 1999-2001. Memoria de título, Escuela de Medicina Veterinaria, Universidad Austral de Chile, Valdivia, Chile.

Alves D, B McEwen, M Hazlett, G Maxie, N Anderson. 1996. Trends in bovine abortions submitted to the Ontario Ministry of Agriculture, Food and Rural Affairs, 1993-1995. Can Vet J 37, 287-288.

Anderson M. 1994. Protozoal causes of reproductive failure in domestic ruminants. Vet Clin North Am Food Anim Pract: Diagnosis of abortion 10, 439-452.

Anonymous. 1986. California livestock statistics. California Crop and Livestock Reporting Service, Department of Food and Agriculture, Sacramento, CA, Pp 10. (Original no consultado, citado por: Thurmond M, J Picanso. 1990. A surveillance system for bovine abortion. Prev Vet Med 8, 41-53).

Barr B, M Anderson, P Blanchard, B Daft, H Kinde, P Conrad. 1990. Bovine fetal encephalitis and myocarditis associated with protozoal infections. Vet Pathol 27, 354-361.

Barr B, M Anderson. 1993. Infectious diseases causing bovine abortion and fetal loss. Vet Clin North Am Food Anim Pract 9, 343-368.

Bartels C, G van Schaik, J Veldhuisen, B van de Borne, W Wouda, T Dijkstra. 2006. Effect of Neospora caninum-serostatus on culling, reproductive performance and milk production in Dutch dairy herds with and without a history of Neospora caninum- associated abortion epidemics. Prev Vet Med 77, 186-198.

Bartels C, I Huinink, M Beiboer, G van Schaik, W Wouda, T Dijkstra, A Stegeman. 2007. Quantification of vertical and horizontal transmission of Neospora caninum infection in Dutch dairy herds. Vet Parasitol 148, 83-92.

Bercovich Z. 1998. Maintenance of Brucella abortus-free herds: a review with emphasis on the epidemiology and problems in diagnosing brucellosis in areas of low prevalence. Vet Quart 20, 81-169.

Bigras-Poulin M, A Meek, S Martin. 1990. Interrelationships among health problems and milk production from consecutive lactations in selected Ontario Holstein cows. Prev Vet Med 8, 15-24.
Bolin C. 1998. Clinical signs, diagnosis, and prevention of bovine leptospirosis. The Bovine Practitioner 33, 50-55.

Campero C, D Moore, A Odeon, A Cipolla, E Odriozola. 2003. An etiology of bovine abortion in Argentina. Vet Res Commun 27, 359-369.

Carpenter T. 2001. Financial considerations of the sets technique in animal-disease surveillance. Prev Vet Med 48, 155-165.

Casteel S. 1997. Reproductive toxicology. In: Youngquist R (ed). Current Therapy in Large Animal Theriogenology. WB Saunders Company, University of Missouri, USA, Pp 392-399.

Celedón M, J Carbonell, L Ibarra, J Pizarro. 1998. Detección de bovinos portadores e inmunotolerantes al virus de la DVB en predios lecheros de la región metropolitana de Chile. Arch Med Vet 30, 125-132.

Cleves M, W Gould, R Gutiérrez. 2004. An introduction to survival analysis using Stata. Stata Press. Texas, USA.

Dohoo I, S Martin. 1984. Disease, production and culling in Holstein Friesian cows. III. Disease and production as determinants of disease. Prev Vet Med 2, 671-690.

Dohoo I, W Martin, H Stryn. 2003. Veterinary epidemiology research. AVC Inc., University of Prince Edward Island, Canada.

Donald H. 1943. Heath during pregnancy in dairy cows. Vet Rec 55, $297-$ 298. (Original no consultado, citado por: Sturman H, E Oltenacu, R Foote. 2000. Importance of inseminating only cows in estrus. Theriogenology 53, 1657-1667).

Dubey J. 1999. Neosporosis in cattle: biology and economic impact. J Am Vet Med Assoc 214, 1160-1163.

Dubovi E. 1994. Impact of bovine viral diarrhea virus on reproductive performance in cattle. Vet Clin North Am. Food Anim Pract: Diagnosis of abortion 10, 503-514.

Ellis W. 1994. Leptospirosis as a cause of reproductive failure. Vet Clin North Am: Food Anim Pract 10, 463-477.

Erb H, S Martin, N Ison, S Swaminathan. 1981․ Interrelationships between production and reproductive diseases in Holstein cows. Conditional relationships between production and disease. J Dairy Sci 64, 272-281.

Erb H, S Martin, N Ison, S Swominathan. 1981 ${ }^{\text {b }}$. Interrelationships between production and reproductive diseases in Holstein cows. Path analysis. J Dairy Sci 64, 282-289.

Erb H, R Smith, P Oltenacu, C Guard, R Hillman, P Powers, M Smith, $\mathrm{M}$ White. 1985. Path model of reproductive disorders and performance, milk fever, mastitis, milk yield and culling in Holstein cows. J Dairy Sci 68, 3337-3349.

Fetrow J, D McClary, R Harman, K Butcher, L Weaver, E Studer, J Ehrlch, W Etherington, W Guterbock, D Klinborg, J Reneau, N Williamson. 1990. Calculating selected reproductive indices: recommendations of the American Association of Bovine Practitioners. J Dairy Sci 73, 78-90.

Forar A, J Gay, D Hancock. 1996. The frequency of edemic fetal loss in dairy cattle: a review. Theriogenology 43, 989-1000.

Galleguillos H, A Rivera, J Herrera, L Espejo, P Méndez. 2005. La atención de denuncias de enfermedades animales durante el período 1999-2004. Boletín Veterinario Oficial. Servicio Agrícola y Ganadero, División de Protección Pecuaria 3, 1-27.

García-Ispierto I, F López-Gatius, G Bech-Sabat, P Santolaria, J Yaniz, C Nogareda, F De Rensis, M López-Béjar. 2007. Climate factors affecting conception rate of high producing dairy cows in northeastern Spain. Theriogenology 67, 1379-1385.

Gatica R. 1996. Vaca Repetidora y mortalidad embrionaria. XXIV Jornadas de Buiatría, Paisandú, Uruguay, Pp 11-16.

Givens D. 2006. A clinical, evidence-based approach to infectious causes of infertility in beef catlle. Theriogenology 66, 648-654.

Giri S, P Emau, J Cullor, G Stabenfeldt, M Bruss, R Bondurandt, B Osborne. 1990. Effect of endotoxin and circulating levels of eicosanoids progesterone, cortisol, glucosa and lactic acid, and abortion in pregnant cows. Vet Microbiol 21, 211-231.

Greenland S, J Robins. 1986. Identifiability, exchangeability, and epidemiological confounding. Int J Epidemiol 15, 413-419. 
Gröhn Y, H Erb, Ch Mc Culloch, H Saloniemi. 1990. Epidemiology of reproductive disorders in dairy cattle: associations among host characteristics, disease and production. Prev Vet Med 8, 25-39.

Hansen P. 1997. Effects of environment on bovine reproduction. In: Youngquist R (ed). Current Therapy In Large Animal Theriogenology. WB Saunders Company, University of Missouri, Missouri, USA, Pp 403-415.

Hanson T, F Bedrick, W Johnson, M Thurmond. 2003. A mixture model for bovine abortion and foetal survival. Statistics in Medicine 22, 1725-1739.

Hassan L, H Mohamed, O McDonough, R González. 2000. A CrossSectional study on the prevalence of Listeria monocytogenes and Salmonella in New York Dairy herds. J Dairy Sci 83, 24412447.

Houe H. 1995. Epidemiology of bovine viral diarrhea virus. Vet Clin North Am Food Anim Pract 11, 521-547.

Hovingh E. 2002. Abortions in dairy cattle II. Diagnosing and preventing abortion problems. Virginia Cooperative Extention Publication 404, 289. Regional College of Veterinary Medicine, Virginia Tech, USA.

Jamaluddin A, J Case, D Hird, P Blanchard, J Peauroi, M Anderson. 1996. Dairy Cattle Abortion in California: evaluation of Diagnostic Laboratory Data. J Vet Diagn Invest 8, 210-218.

Kirkbridge C. 1990. Laboratory Diagnosis of Livestock Abortion. $3^{\text {rd }}$ ed. Iowa State University Press. Iowa, USA.

Kirkbridge C. 1992. Etiologic agents detected in a 10-year study of bovine abortions and stillbirths. $J$ Vet Diagn Invest 4, 175-180.

Kleinbaum D. 1996. Survival Analysis: A self learning text. Statistics in the Health Sciences. Springer. New York, USA.

Lemire G, P Stalheim, M Lemire, M Tiemann, L Verdon. 1993. Monitoring pregnancy losses in small dairy herds. Can Vet J 34, 33-35.

Lertora J, E Paredes, G Reinhardt, A Alberdi. 2003. Inmunohistoquímica en biopsias de piel tratadas con proteína $\mathrm{K}$ y microondas para el diagnóstico en animales persistentemente infectados con el virus diarrea viral bovina. Arch Med Vet 35, 23-36

Lindberg A, S Alenius. 1999. Principles for eradication of bovine virus diarrhoea virus infections in cattle populations. Vet Microbiol 64,197-222.

Lindberg A. 2002. Epidemiology and eradication of bovine virus diarrhea virus infections. PhD Thesis, Swedish University of Agricultural Sciences, Uppsala, Sweden.

López-Gatius F, J Santolaria, J Yaniz, J Rutllant, M López-Béjar. 2002. Factors affecting pregnancy loss from gestation day 38 to 90 in lactating dairy cows from a single herd. Theriogenology 57, 1251-1261.

López-Gatius F, M Pabón, S Almería. 2004. Neospora caninum infection does not affect early pregnancy in dairy cattle. Theriogenology 62 , 606-613.

Maldonado G, S Greenland. 1993. Interpreting model coefficients when the true model form is unknown. Epidemiology Resources $4,310-318$

Markusfeld O. 1996. Epidemiology of bovine abortions in Israeli dairy herds. Prev Vet Med 31, 245-255.

Martin S, A Aziz, W Sandals, R Curtis. 1982. The association between clinical disease, production and culling of Holstein Friesian cows. Can J Anim Sci 62, 633-640.

McAllister M, J Dubey, D Lindsay, W Jolley, R Wills, A McGuirre. 1998. Dogs are definitive host of Neospora caninum. Int J Parasitol 28, 1473-1478

McGowan M, P Kirkland. 1995. Early reproductive loss due to bovine pestivirus infection. Br Vet J 151, 262-269.

Meléndez P, A Donovan. 2003. Herd-level ELISA seroprevalence of bovine viral diarrhea antibodies in bulk-tank milk in Chilean dairy herds. Prev Vet Med 60, 237-241.

Miller R. 1986. Bovine Abortion. In: Morrow D (ed). Current Therapy In Theriogenology. WB Saunders Company, Michigan State University, Michigan, USA.
Moennig V, B Liess. 1995. Patogenesis of intrauterine infections with bovine viral diarrhea virus. Vet Clin North Am Food Anim Pract $11,477-487$.

Moore D, W Overton, R Chebel, M Truscott, R BonDurant. 2005. Evaluation of the factors that affect embrionic loss in dairy cattle. $J$ Am Vet Med Assoc 226, 1112-1118.

Muñoz-Zanzi C, S Hietala, M Thurmond, O Jonson. 2003. Quantification, risk factors, and health impact of natural congenital infection with bovine viral diarrhea virus in dairy calves. Am J Vet Res 64, 358-365.

OIE, World Organization for Animal Health. 2007. Terrestrial Animal Health Code. $16^{\text {th }}$ ed. World Organization for Animal Health, Paris, France. Pp 83-87.

Otte M, T Ravenborg, K Hüttner. 1995. A pilot study of elevated abortion and stillbirth ratios in cattle in the foothills of the eastern plains of Colombia. Prev Vet Epidemiol 22, 103-113.

Pabón M, F López-Gatius, I García-Ispierto, G Bech-Sabat, C Nogareda, S Almería. 2007. Chronic Neospora caninum infection and repeat abortion in dairy cows: A 3 year study. Vet Parasitol 147, 40-46.

Paré J, M Thurmond, S Hietala. 1997. Neospora caninum antibodies in cows during pregnancy as a predictor of congenital infection and abortion. J Parasitol 83, 82-87.

Paredes E, M Pradenas, A Arancibia. 2002. Principales causas de aborto bovino diagnosticadas en el Instituto de Patología Animal de la Universidad Austral de Chile; período 1990-1999. XII Congreso Chileno de Medicina Veterinaria, Chillán, Chile.

Paredes E, M Moroni. 2005. Principales causas de abortos diagnosticadas en el período 2003-2005 en fetos bovinos examinados en el Instituto de Patología Animal, Universidad Austral de Chile. XII Congreso Latinoamericano de Buiatría, Valdivia, Chile, Pp 148-149.

Patitucci A, M Pérez, C Luders, M Ratto, A Dumont. 1999. Evidencia serológica de infección por Neospora caninum en rebaños lecheros del sur de Chile. Arch Med Vet 31, 215-218.

Patitucci A, M Pérez, K Israel, M Rozas. 2000. Prevalencia de anticuerpos séricos contra Neospora caninum en dos rebaños lecheros de la IX Región de Chile. Arch Med Vet 32, 209-214.

Ramírez M, S Ernst, F Elvinger, A Rivera, C Rosenfeld. 2002. Respuesta serológica y tiempo de saneamiento en rebaños bovinos con brucelosis vacunados con Cepa 19 o Cepa RB-51; X Región, Chile. Arch Med Vet 34, 213-220.

Reimers T, R Smith, S Newman. 1985. Management factors affecting reproductive performance of dairy cows in the northeastern United States. J Dairy Sci 68, 963-972.

Reinhardt G, L Carrasco, N Tadich, S Riedemann. 2001. Comparación entre dos técnicas de diagnóstico para Diarrea Viral Bovina (DVB) en 50 predios de la X Región, Chile: Seroneutralización y enzimoinmunoensayo indirecto (ELISA-I). Arch Med Vet 33, 173-183.

Reinhardt G, S Riedemann, N Tadich. 2002. Muestreo predial pequeño para predecir una infección activa por virus diarrea viral bovina (VDVB) en planteles lecheros de la X Región de Chile. Arch Med Vet 34, 97-101.

Risco C, G Donovan, J Hernández. 1999. Clinical mastitis associated with abortion in dairy cows. J Dairy Sci 82, 1684-1689.

Rivera G. 2001. Causas frecuentes de aborto bovino. Rev Investig Vet Perú 12, 117-122.

Roche J, M Bolandl, T McGeady. 1981. Reproductive wastage following artificial insemination of heifers. Vet Rec 109, 41-404.

Rothman K. 2002. Epidemiology, an introduction. Oxford University Press, New York, USA.

Sanz A, A Bruñes, D Villalba. 2004. Influence of management and nutrition on pospartum interval in Brown Swiss and Pirenaica cows. Livest Prod Sci 86, 179-191.

Sickles S, J Kruze, R González. 2000. Aislamiento de Mycoplasma bovis en muestras de leche de estanque en rebaños lecheros del sur de Chile. Arch Med Vet 32, 235-240.

Stevenson J. 1997. Clinical reproductive phisiology of the cow. In: Youngquist R (ed). Current Therapy In Large Animal Theriogenology. WB Saunders Company, University of Missouri, Missouri, USA. 
Sturman H, E Oltenacu, R Foote. 2000. Importance of inseminating only cows in estrus. Theriogenology 53, 1657-1667.

Thrusfield M. 1995. Veterinary epidemiology. $2^{\text {nd }}$ ed. The University Press, Cambridge, United Kingdom.

Thurmond M, J Picanso. 1990. A surveillance system for bovine abortion. Prev Vet Med 8, 41-53.

Thurmond M, J Picanso, C Jameson. 1990 . Considerations for use of descriptive epidemiology to investigate fetal loss in dairy cows. $J$ Am Vet Med Assoc 197,1305-1312.

Thurmond M, J Picanso, S Hietala. 1990 b . Prospective serology analysis in diagnosis of dairy cows abortion. J Vet Diagn Invest 2, 274-282.

Thurmond M, J Picanso. 1993. Fetal loss associated with palpation per rectum to diagnose pregnancy in cows. J Am Vet Med Assoc 203, 432-435.

Thurmond M, P Blanchard, M Anderson. 1994. An example of selection bias in submissions of aborted bovine fetuses to a diagnostic laboratory. J Vet Diagn Invest 6, 269-271.

Thurmond M, W Johnson, C Muñoz-Zanzi, C Su, S Hietala. 2002. A method of probability diagnostic assignment that applies bayes theo- rem for use in serologic diagnostics, using an example of Neospora caninum infection in cattle. Am J Vet Res 63, 318-325.

Thurmond M, A Branscum, W Johnson, E Bedrick, T Hanson. 2005. Predicting the probability of abortion in dairy cows: a hierarchical Bayesian logistic-survival model using sequential pregnancy data. Prev Vet Med 68, 223-239.

Wolfgang D. 2003 ${ }^{\text {a }}$ Investigating abortions in cattle: part III Interpretation of results associated with bacteria, Dairy \& Animal Science Dairy Digest, DAS 66, Penn State College of Agricultural Sciences, USA.

Wolfgang D. $2003^{\mathrm{b}}$. Investigating abortions in cattle: part IV Interpretation of results associated with viruses, Dairy \& Animal Science Dairy Digest, DAS 68, Penn State College of Agricultural Sciences, USA.

Wouda W, A Moen, H Schukken. 1998. Abortion risk in progeny of cows after a Neospora caninum epidemic. Theriogenology 43, 1311-1316.

Zamora J, S Riedemann. 1999. Animales silvestres como reservorios de leptospirosis en Chile. Una revisión de los estudios efectuados en el país. Arch Med Vet 31, 151-156. 